\title{
A Phase and Frequency Locking Loop for Engine Air-Fuel Ratio Control
}

\author{
Sharon Liu, Kenneth P. Dudek, Robert D. Shafto \\ sharon.2.liu@gm.com
}

\begin{abstract}
In the spirit of the recent articles in [5], featuring Classical Controls Revisited, a Phase-Lock Loop concept is applied to an automotive air-fuel ratio closed-loop control system. This classical solution is analyzed, simulated, and experimentally implemented in a vehicle. The results demonstrate that the automobile engine air-fuel ratio system can be controlled to robustly meet emissions and driving performance requirements by using a simple classical control methodology with feedback from the common switching oxygen sensor.
\end{abstract}

\section{Introduction}

A comprehensive survey of automotive engine control systems is available as a tutorial from 2005, [14]. In particular, the tutorial exemplifies air-fuel ratio $(\mathrm{A} / \mathrm{F})$ control with torque control, reviewing a number of approaches used by different researchers. Due to the plethora of linear model-based control techniques, a good number of references from [14] and elsewhere, choose to employ wide-range oxygen sensors for the feedback measurement. For example, [11] facilitates the use of an $H_{\infty}$ control law design by linearizing the system model and closing the loop with the linear wide-range oxygen sensor; [2] first demonstrates successful transient air-fuel ratio control with an estimator built in event space, using linear feedback measurements; [4] produced an impressively robust predictive observer air-fuel ratio control system requiring the linear oxygen sensor for feedback information; and [9] uses the linear oxygen sensor to venture into constructing semi-positive definite control lyapunov functions to nonlinearly control both the fuel and air such that torque and air-fuel ratio specifications can be met. There are potentially a variety of alternatives to the wide-range oxygen sensor measurement for closing the loop on the air-fuel ratio system, such as using individual cylinder pressure sensors to provide peak pressure and its associated crank angle per firing to sustain engine air-fuel ratio operations that may not be stoichiometric. But for gasoline automotive engines that are required to always run at stoichiometry in order to capitalize on the maximum efficiency of the three-way catalytic conversion, to ensure meeting emissions mandates, a binary or switching oxygen sensor is often the common option.

According to [4], while the wide-range linear oxygen sensor enables so much A/F closed-loop robustness as that the system can reject throttle variations at $1 \mathrm{~Hz}$ between $25-45 \%$, switching sensors have faster response. Also according to [4] the delay of the air-fuel information from the induction and fueling event is a significant drawback, which effects any choice of Exhaust Gas Oxygen sensor (EGO) located downstream from the cylinder exhaust ports. But this delay is known or can be expected in general to be approximately 2 engine cycles per [17]. Additionally, [3] shows that the closed-loop air-fuel ratio with a switching oxygen sensor can be made to track a dithering air-fuel reference, regardless of the choice of waveforms; this seems to indicate some robustness. Finally, [8] dithers the throttle air intake up to $\pm 5 \%$ at approximately 1-2 $\mathrm{Hz}$ as part of an air-fuel ratio estimator-control design with claims of meeting acceptable driving standards.

In light of the background literature and discussions in [5], a digitally implemented closed-loop control law that is based on the Phase-Lock Loop technique is modified for the engine air-fuel ratio system. Because the air induction system can be quite complex with modern engines using electronic throttle control (ETC), exhaust gas recirculation (EGR), as well as, variable valve timing (VVT), and given results from [12] that the switching pre-catalyst oxygen sensor can be successfully used to compensate the fuel for transient air-fuel ratio objectives, the single inputsingle output (SISO) system under consideration actuates by adjusting the fuel and feeding back the switching oxygen $\left(\mathrm{O}_{2}\right)$ sensor measurement. A SISO classical approach is appealing because it can be simply built for implementation as well as analysis, without modeling the relatively complex engine induction to exhaust emissions sys- 
tem.

Remarkably, the switching $\mathrm{O}_{2}$ sensor is nonlinear and sometimes causes unobservability of the system behavior. The switching $\mathrm{O}_{2}$ sensor provides useful information in only 3 ways; when there is a switch between high and low voltage the engine ran approximately stoichiometrically, when the sensor reads high voltage the engine ran rich, and when the sensor reads low voltage the engine ran lean. But if efficient three-way catalytic conversion to process a conventional gasoline engine exhaust is desired and, as such, requires the engine to run stoichiometrically, then having a regularly or periodically switching measurement suffices. Notably, [10] takes advantage of just this occasion to develop a sliding-mode observer for controlling both the air and fuel while [17] elaborates with a Gaussian Network sliding mode control.

The ubiquitous architecture in [1] for the airfuel ratio control problem is shown in Figure 2 with some adjustments in detail. Controlling the closed-loop system such that the feedback measurement synchronizes to the oscillatory input is like the Phase-Lock Loop shown in section 2, with a controlled oscillator automatically synchronizing to a reference or carrier signal. From [6], analyses show that the Phase-Lock Loop (PLL) is essentially linear in the operations of interest despite the oscillating amplitude. This method of using the switching sensor information allows the nonlinear sensor to be treated linearly.

In the section 2 we review the analogue phaselock loop in order to indicate the modifications necessary to accommodate the automotive airfuel ratio feedback control system. This is followed by a description of the modified algorithm simulated and experimentally implemented in a vehicle, in section 3 . In section 4 some experimental vehicle results are summarized and a critique is given of the technique employed. Figure 4 and 5 show experimental results which substantiate the viability of the approach.

\section{Review}

The phase-lock loop circuit was and still is used commonly when receiving radio signals [16]. This technique is discussed in [6], including analyses of linear properties and the inevitable nonlinear phenomenon which occurs when the lock breaks or slips and other sundry difficulties. Figure 1 illustrates the schematic for the basic components.

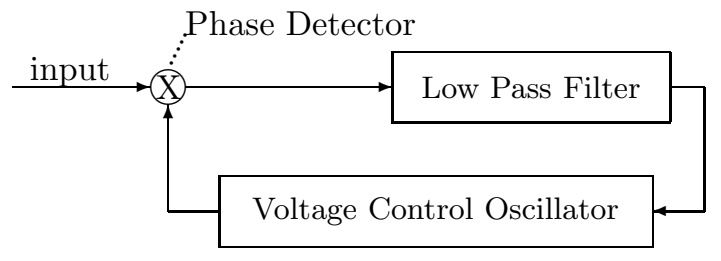

Figure 1: The principle components of an analogue phase-lock loop.

Given a known frequency of the input signal and a Voltage Controlled Oscillator (VCO) that can output approximately the same frequency, the low-pass filtered product of these two signals constructed by the Phase Detector is a phase error compensator that drives the closed-loop circuit to phase-lock. Specifically, if the input is $A_{i} \sin (\omega t)$ and the VCO outputs $A_{o} \sin (\omega t+\phi)$ where $\omega$ is the frequency of operation and $\phi$ is the phase difference being attenuated, $t$ is the time, $A_{i}$ and $A_{o}$ are the input and VCO output amplitudes respectively, then the Phase Detector circuit multiplies its two inputs, $A_{i} \sin (\omega t) A_{o} \sin (\omega t+\phi)$ to output the phase difference with some higher frequency 'noise.'

$$
\frac{A_{i} A_{O}}{2}[\cos (-\phi)-\cos (2 \omega t+\phi)]
$$

An explanation of the VCO and Phase Detector circuits can be found in [7]. The low-pass filter eliminates the high frequency term and the phase error is input to the VCO as a change in voltage that drives its output to synchronize with the reference input.

In the engine air-fuel ratio application, we command a feedforward dither signal of a given frequency as if it were a reference carrier signal and we strive to control the engine system to this frequency as if it were essentially a VCO or $\mathrm{Nu}$ merically/Digitally Controlled Oscillator (DCO) [15] such that the closed-loop we build regulates the $\mathrm{O}_{2}$ sensor switching output effectively as if by locking the engine system in phase with our reference command. However, an engine system is a reluctant oscillator so modifications are necessary to achieve this goal.

\section{Modified application}

The primary requirement for the air-fuel ratio closed-loop system as configured in figure 2 to be considered a phase-lock loop is that the airfuel ratio system must be controlled to oscillate about stoichiometric engine operation, which depends on the switching $\mathrm{O}_{2}$ sensor measurement. 


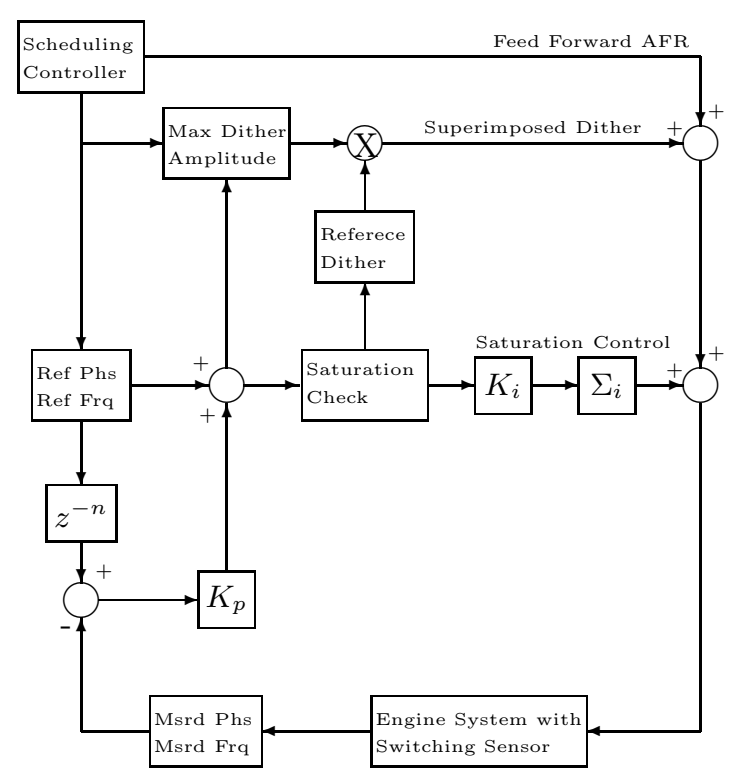

Figure 2: The closed-loop schematic for a PhaseLock Loop implementation on an engine air-to-fuel ratio system with the precatalyst switching $\mathrm{O}_{2}$ sensor for the feedback measurement.

This seems a self-referencing dilemma that can be resolved, luckily, by the fact that the reference signal can be changed with the oscillation control and, hence, can be used as an additional degree of freedom. This is commonly known in communications studies as a variable frequency oscillator (VFO).

Since the engine is controlled digitally, and the engine naturally runs at discrete-time combustion events, the algorithm development follows the digital phase-lock loop, that [15] and [16] also address. Commensurate with the digital phaselock loop methodology, the analogue $\mathrm{O}_{2}$ sensor switching signal is quantized to readily compute threshold crossings for determining the frequency and phase of switches. The reference periodic input is constructed as a pulse-train. The comparison between the quantized $\mathrm{O}_{2}$ output and the reference input gives a frequency and phase error in terms of integer number of samples.

In the linear range of the algorithm, when the Saturation Check block outputs only to the Reference Dither block, for analysis, figure 2 can be reduced to a slightly modified phase-lock loop structure due to the feedforward input, as shown in figure 3 .

The resulting transfer function from the delayed reference to the DCO, or engine sensor, output

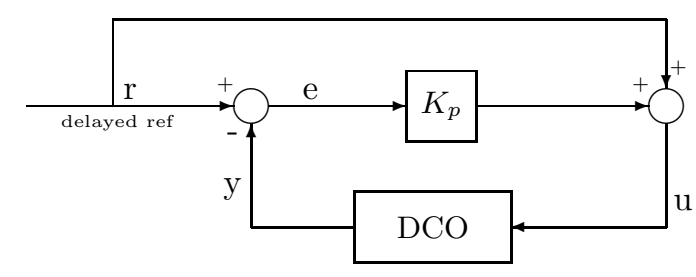

Figure 3: Representation of the the Linear Range operation when the Saturation Check inputs nothing to the integrator.

is easily found.

$$
\begin{aligned}
e & =r-y \\
y & =\mathrm{D} C O u \\
u & =r+K_{p} e \\
\frac{y}{r} & =\frac{\mathrm{DCO}\left(1+K_{p}\right)}{1+K_{p} \mathrm{DCO}}
\end{aligned}
$$

The choice of reference frequency is scheduled for different operations such that the engine system responds reasonably like a variable frequency oscillator. The DCO can be represented as a second order oscillator with a discrete transfer function

$$
\frac{1}{z^{2}+1}
$$

Let $K_{p}$ be a generic classical linear compensator with a PID or lead-lag transfer function. Then the switching sensor output $y$ to the reference switching $r$ can be expressed as

$$
\frac{y}{r}=\frac{1+K_{p}}{z^{2}+1+K_{p}}
$$

Under this linear range, the full set of linear systems control theory can be exercised to achieve the performance desired up to the physical limitations of the system response. For even $K_{p}=$ 1, a simulation in Mathworks ${ }^{\mathrm{TM}}$ Simulink ${ }^{\mathrm{TM}}$ can verify that the closed-loop system follows the reference pulse-train for frequencies greater than twice the sampling frequency, as is usually required.

If the phase or frequency errors are sufficiently large that for all practical purposes, the sensor is not switching, then the Saturation Check block augments the linear controller with integration action transformed to correcting the amplitude of the air-fuel ratio. This portion of the algorithm is akin to a bang-bang control approach with incremental, self-tuning time-varying control limits. In particular, once saturation is reached, for each sample time saturation continues, the integrated phase or frequency error applied to the amplitude indicates the magnitude for the air-fuel ratio 

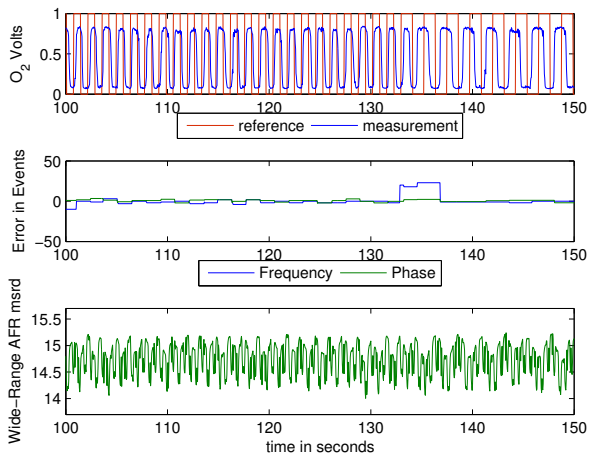

Figure 4: Different frequencies tracked while idling. The top plot shows the reference pulse train mostly followed by the measured oxygen sensor quantized voltage output. The middle plot counts the number of events different between the measurement from the reference signal in both inverse frequency and phase. The last plot shows the measurement from a development wide-range air-fuel ratio sensor indicating that the switching sensor information is reasonably reliable.

deviation from stoichiometry has not been compensated. The drawback to this approach is that once the sensor switches again, the integrated error would continue to compensate for at least the period of the system delay, potentially causing the air-fuel ratio system to saturation in the other direction. This is an inevitable consequence of both the information delay to the sensor, and the lack of system detectability outside the linear range of algorithmic operations. Whether linear operations can resume depends on the air-fuel ratio disturbance to diminish or reach steady state over time. However, not attaining the linear operating conditions does not necessarily preclude effectively controlling for emissions as long as the saturation compensation achieves sensor output switching albeit irregularly.

\section{Results}

The control algorithm described in sections 2 and 3 is implemented, with once per cycle sample rate, in a vehicle using commercially available rapid prototyping hardware and software on a 4-cylinder application. The plots in figure 4 demonstrate that using a simple proportional gain for $K_{p}$ is sufficient to allow the quiescent system to track variable frequencies with relatively quick recovery after frequency command changes, as suggested by the simple Mathworks ${ }^{\mathrm{TM}}$ simulations mentioned in section 3.
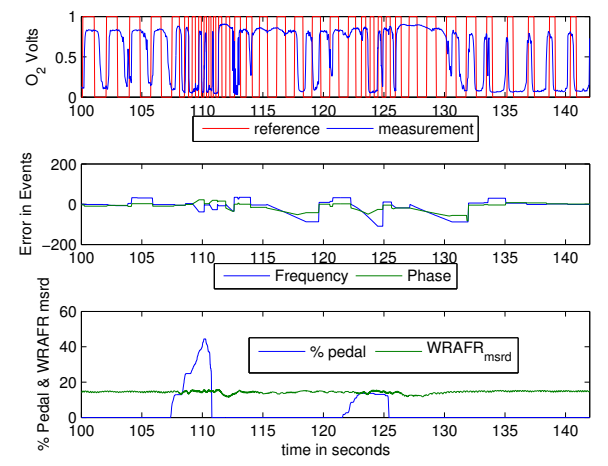

Figure 5: A fixed frequency is commanded while driving. The top plot shows the reference pulse train not tracked by the oxygen sensor during transients. The middle plot is the error incurred during saturation control. The bottom plot shows that the throttle input are much faster than the frequency commanded and recovery to the linear control regime, which can guarantee tracking, is not easily attain if new disturbances arise too soon.

Once the system begins to operate other than at idle, we see from figure 5 that a more sophisticated feedback compensator may be necessary. In addition to the long system delay from the air-fuel ratio input to reach the sensor, time is required to ascertain the measured frequency and phase. Specifically, the period commanded to produce the desired switching frequency must be over sufficiently large number of events to ensure the measured phase and all computed errors are correct and do not become too noisy, especially at higher engine speeds. Therefore, at the very least, added to the delay from the sensor measurement is the delay of the length of the commanded period; only after this time can an error be legitimately computed. Such system latency may pose a significant challenge regardless of choice in control laws. Notably, many engine operating transients occur in much shorter timeframes and will be missed by the algorithm while the linear range may adequately compensate the disturbance. The delayed saturation controller response can be seen as detrimental to the ensuing recovery because the integrator effectively winds-up. Another air-fuel ratio transient excursion before the controller recovers or unwinds may prevent the system from reaching its linear region of control and result in a lot of control effort for little to no regular dither tracking. 


\section{Conclusions}

A classical phase-lock loop concept is revised for the engine air-fuel ratio closed-loop system which uses a switching pre-catalyst oxygen sensor. The technique converts the nonlinear sensor measurement such that it and the entire system can be controlled linearly for some significant portions of engine operating conditions. While this achieves excellent results for quiescent operating conditions, the performance can be greatly improved under transients when the system delays and algorithm induced delays prevent effective control action required to regulate the engine to the variable commanded oscillations.

\section{References}

[1] Buckland, Julia, Jeffrey A. Cook, Automotive Emissions Control, Proceedings of the 2005 American Control Conference, June 8-10, 2005, Portland, OR, USA, pp. 3290-3295.

[2] Chang, Chen-Fang, Nicholas P. Fekete, and J. David Powell, Engine Air-Fuel Ratio Control Using an Event-based Observer, SAE Technical Paper 930766, Society of Automotive Engineers, Warrendale, Pa., 1993.

[3] Chang, Chen-Fang, Nicholas P. Fekete, Alois Amstutz, and J. David Powell, Air-fuel Ratio Control in Spark-Ignition Engines Using Estimation Theory, IEEE Transactions on control Systems Technology, Vol. 3, No. 1, March 1995, pp. 22-31.

[4] Chevalier, Alain, Christian Winge Vigild, Elbert Hendricks, Predicting the Port Air Mass Flow of SI Engines in Air/Fuel Ratio Control Applications, SAE Technical Paper 2000-010260, Society of Automotive Engineers, Warrendale, Pa., 2000.

[5] Davidson, Daniel E. and Jie chen, Classical Control Revisited, Control Systems Magazine, Vol 27, No. 1, February 2007, pp.20-21.

[6] Gardner, Floyd M., Phaselock Techniques, John Wiley \& Sons Inc (1967).

[7] Horowitz, Paul and Winfield Hill, The Art of Electronics, 2nd ed., Cambridge University Press (1989).

[8] Jones, V.K., Brian A. Ault, Gene F. Franklin, and J. David Powell, Identification and Air-Fuel Ratio Control of a Spark-Ignition Engine, IEEE Transactions on control Systems Technology, Vol. 3, No. 1, March 1995, pp. 14-21.

[9] Kang, Jun-Mo and J.W. Grizzle, Nonlinear Control for Joint Air and Fuel Management in a SI Engine, Proceedings of the 1999 American
Control Conference, June 1999, San Diego, CA, USA, pp. 3290-3295.

[10] Kim, Yong-Wha, Giorgio Rizzoni, and Vadim Utkin, Automotive Engine Diagnosis and Control via Nonlinear Estimation, Control Systems Magazine, Vol 18, No. 5, October 1998, pp.84-99.

[11] Mianzo, Larry, Huei Peng, and Ibrahim Haskara, Transient Air-Fuel Ratio $H_{\infty}$ Preview Control of a Drive-by-Wire Internal Combustion Engine, Proceedings of the 2001 American Control Conference, June 25-27, 2001, Arlington, VA, USA, pp. 2867-2871.

[12] Moraal, P.E., Adaptive Compensation of Fuel Dynamics in an SI Engine using a Switching EGO Sensor, Proceedings of the 34th Conference on Decision and Control, Dec 13-15, 1995, New Orleans, LA, USA, vol. 1, pp. 661-666.

[13] Onder, Christopher H. and Hans P. Geering, Model-Based Multivariable Speed and Air-toFuel Ratio Control of an SI Engine, SAE Technical Paper 930859, Society of Automotive Engineers, Warrendale, Pa., 1993.

[14] Sun, Jing, Ilya Kolmanovsky, Jeffrey A. Cook, Julia H. Buckland, Modeling and Control of Automotive Powertrain Systems: A Tutorial, Proceedings of the 2005 American Control Conference, June 8-10, 2005, Portland, OR, USA, pp. 3271-3283.

[15] Wikipedia contributors, Numericallycontrolled oscillator, Wikipedia, The Free Encyclopedia, http://en.wikipedia.org/w/ index . php?title=Numerically-controlled oscillator\&oldid=146135676, (accessed July $30,2007)$.

[16] Wikipedia contributors, Phase-locked loop, Wikipedia, The Free Encyclopedia, http://en.wikipedia.org/w/index.php? title=Phase-locked_loop\&oldid=146638337, (accessed July 30, 2007).

[17] Won, Mooncheol, Seibum B. Choi, and J.K. Hedrick, Air-to-Fuel Ratio Control of SparkIgnition Engines Using Gaussian Netwoek Sliding Control, IEEE Transactions on control Systems Technology, Vol. 6, No. 5, September 1998, pp. 678-687.

[18] Yoon, Paljoo, Seungbum Park, and Myoungho Sunwoo, Closed-Loop Control of Spark Advance and Air-Fuel Ratio in SI Engines Using Cylinder Pressure, SAE Technical Paper 200001-0933, Society of Automotive Engineers, Warrendale, Pa., 2000. 